\title{
Trajectories of adjustment disorder symptoms in post-treatment breast cancer survivors
}

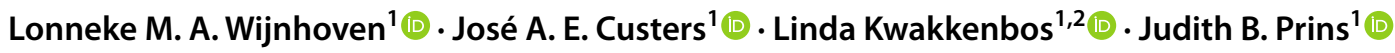

Received: 26 April 2021 / Accepted: 31 December 2021 / Published online: 12 January 2022

(c) The Author(s) 2022

\begin{abstract}
Objective Breast cancer survivors (BCS) may experience problems to adjust to their situation after cancer treatment completion. In case of severe distress, an adjustment disorder (AD) might develop. This study investigates the course of AD symptoms during 1 year and its predictors in BCS up to 5 years post-treatment.

Methods BCS completed the Hospital Anxiety and Depression Scale (HADS) at baseline, 3, 6, and 12 months. HADS total scores were defined as no mental disorder (MD) symptoms $(\leq 10)$, AD symptoms (11-14), and any other MD symptoms $(\geq 15)$. Over the course of four assessments, symptom trajectories were a priori defined as no MD symptoms, AD symptoms, fluctuating AD symptoms below and above cut-offs, or any other MD symptoms. Complementary, latent class growth analysis (LCGA) was used to identify data-driven trajectories.

Results Among 293 BCS with complete data, the majority was classified as no MD symptoms (54.4\%), followed by $37.5 \%$ in the fluctuating AD symptoms trajectory. Only 1.4\% had AD symptoms, and 6.8\% had any other MD symptoms. With LCGA $(N=459)$, three trajectories were found: stable no MD symptoms $(58.6 \%)$, stable AD symptoms $(32.9 \%)$, and high increasing any other MD symptoms (8.5\%). Compared to BCS with no MD symptoms, BCS with fluctuating AD symptoms or any other MD symptoms were younger, less able to handle daily activities, and showed more social support discrepancy, neuroticism, and less optimism.

Conclusions Results of our study showed that AD symptoms in BCS up to 5 years post-treatment fluctuate over 1 year. It is thus important to appropriately assess $\mathrm{AD}$ over the course of 5 years post-treatment as $\mathrm{AD}$ symptoms can fluctuate.
\end{abstract}

Keywords Adjustment disorder · Breast cancer survivors · Trajectory analysis · Oncology $\cdot$ Psychology

\section{Background}

Breast cancer is the most prevalent cancer type in women. Improved methods for early cancer detection and innovations in cancer treatment have increased the 5-year survival rate in breast cancer survivors (BCS), which is currently $91 \%$ in The Netherlands [2, 32]. As a consequence, more BCS are dealing with the long-term complications of their cancer treatment, including the psychological burden $[5,14,24$, 38]. When cancer-related distress is severe, an adjustment

Lonneke M. A. Wijnhoven

Lonneke.Wijnhoven@radboudumc.nl

1 Department of Medical Psychology 926, Radboud University Medical Center, Radboud Institute for Health Sciences, PO Box 9101, 6500 HB Nijmegen, The Netherlands

2 Clinical Psychology, Behavioural Science Institute, Radboud University, Nijmegen, The Netherlands disorder (AD) can be diagnosed [10, 39], if symptoms are not solely an exacerbation of a pre-existing mental disorder (MD) and the criteria of another MD are not met. In The Netherlands, reimbursement of psychological interventions for cancer survivors is available if a MD such as an anxiety disorder or major depression is diagnosed. It is currently being investigated whether $\mathrm{AD}$ can be added to the reimbursement scheme.

In the DSM-V, AD has been defined as the presence of emotional and behavioural symptoms in response to an identifiable stressor(s) occurring within 3 months of the onset of the stressor(s). The accompanied distress is out of proportion to normal reactions to the stressor in social or cultural context. After ceasing of the stressor or its consequences, symptoms of $\mathrm{AD}$ resolve within 6 months (Criterion $\mathrm{E}$ ); however, if stressors or its consequences continue, this may result in persistent $\mathrm{AD}$ [3]. After curative cancer treatment, continuous confrontation with stressors is possible due to 
for instance ongoing adjuvant endocrine therapies, imaging, and follow-up appointments as well as long-term consequences of cancer such as fatigue, fear of cancer recurrence, and reduced ability to work. Critics debate that a MD diagnosis based on distress symptoms alone medicalizes problems of living [6] and that the AD diagnosis is unclear in discriminating a MD from a normal stress reaction [10]. More insight in $\mathrm{AD}$ in relation to trajectories of psychological adjustment after cancer is necessary.

Predictors of AD related to cancer have not been thoroughly investigated [9]. In a large mixed cancer sample $(N=2141)$, higher education, having metastases, and being female were identified as predictors for AD [19]. In another study in cancer patients, the more commonly investigated symptom distress was found to be predicted by more neuroticism, and findings on optimism were inconclusive [12].

Among patients with breast cancer, the prevalence of $\mathrm{AD}$ was estimated to be $7.1 \%$ in the acute phase of treatment [30], 38.6\% in the first year post-diagnosis [41], 14.4\% in BCS [29], and $20 \%$ in BCS with a first recurrence of breast cancer [23] when assessed with (semi)structured interviews, the golden standard to diagnose $\mathrm{AD}$ in clinical settings [31]. It is advised to screen patients with cancer for psychosocial problems prior to conducting clinical assessments [34]. Although screening is common for depression [7], anxiety [20], and posttraumatic stress disorder [4], measures focussing on AD, e.g. Adjustment Disorder New Module [28], the Diagnostic Interview Adjustment Disorder [13], and International Adjustment Disorder Questionnaire [36], are mostly used for research purposes. The Hospital Anxiety and Depression Scale (HADS), a commonly used screening questionnaire in cancer survivors, measures emotional distress with symptoms of depression and/or anxiety [43]. Since these symptoms are in line with the diagnostic criteria of $\mathrm{AD}$, the HADS might also be used to screen for $\mathrm{AD}$ symptoms. Several studies have reported that the HADS is sensitive to identifying cases of $\mathrm{AD}$ in patients with cancer $[1,25,33]$.

Understanding the course of AD symptoms over time may help identify BCS who develop persistent AD. Distinct distress trajectories in patients with breast cancer were observed up to 8 months post-diagnosis [18, 22, 26, 27]. Only one study followed BCS up to 4 years [17]. Distress trajectories were identified as stable low (36-80\%), stable high $(9-15.4 \%)[17,18,22,26,27]$, recovery $(5.6 \%$ and $12 \%)[26,27]$, delayed recovery $(7-27 \%)[17,22,26]$, and worsening $(4.5 \%$ and $7.9 \%)$ [22, 27]. One study identified distress trajectories during active treatment $(33.3 \%)$ and during the re-entry and survivorship phase (15.2\%) [18]. Predictors that distinguished trajectories were age [26], physical symptoms [26, 27] at treatment completion [22], satisfaction with medical consultation [26], history of psychiatric illness [27], personal [17] and social resources [17, 27], mastery
[18, 27], optimism [18, 26], neuroticism [18], and benefit finding [27]. Most studies [17, 18, 22, 26] used a growth mixture modelling approach to determine trajectories from a data-driven point of view, and one study used cut-off scores from a clinical point of view [27].

The primary aim of this study was to detect trajectories of $\mathrm{AD}$ symptoms during 1 year using the HADS in BCS, using both clinically relevant cut-off scores and a data-driven growth modelling approach. The secondary aim was to identify predictors for distinct trajectories.

\section{Methods}

\section{Participants and procedure}

Physicians invited 1205 BCS from three hospitals in The Netherlands to participate with an information letter. Eligible participants were cancer-free, $\geq 18$ years old, with stages I-III breast cancer treated with curative intent, who finished primary cancer treatment in the past 5 years, and were able to complete questionnaires in Dutch. BCS currently on hormonal therapy or treatment with specific antibodies (trastuzumab) were also eligible. After informed consent, participants received a questionnaire booklet (paper-and-pencil) or email with a link to a secured online system. Questionnaires were sent upon enrolment and after 3, 6, and 12 months.

\section{Demographic, clinical, and psychosocial measures}

Participants completed socio-demographic variables (e.g. age and employment status) and self-reported clinical variables (e.g. type of treatment and time since diagnosis) in the baseline questionnaire.

$A D$ symptoms were assessed using the HADS, a 14-item questionnaire with subscales Anxiety (HADS-A, 7 items) and Depression (HADS-D, 7 items) [37]. Items are scored on a scale (range $0-3$ ), resulting in subscale $(0-21)$ and HADS total (0-42) scores. For cancer survivors [43], cut-off scores were identified of HADS total $\geq 10$ or 11 for screening for MDs (sensitivity 0.80 ; specificity 0.74 ) and $\geq 15$ for screening for depression (sensitivity 0.87 ; specificity 0.88 ).

Social support was measured with the Social Support List-Discrepancies (SSL-D), a 34-item questionnaire (4-point Likert scale). The SSL-D measures the perceived discrepancy between the amount of received social support and the desired amount of social support [42], further referred to as social support discrepancy. A higher total score of SSL-D (range 34-102) indicates more social support discrepancy. The test-retest reliability is 0.85 and a Cronbach's alpha of 0.95 [42].

Optimism was measured with the Life Orientation Test (LOT), a 12-item questionnaire (5-point Likert scale, 
$0=$ "strongly disagree" to $4=$ "strongly agree") reflecting generalized optimism versus pessimism. Higher total scores (range 0-32, 4 filler items excluded) indicated more optimism. LOT-total Cronbach's alpha is 0.76, and test-retest reliability is 0.79 [35].

Neuroticism was measured using the Big Five Inventory (BFI) [16], a 44-item questionnaire designed to measure the Big Five factor structure of personality (5-point Likert scale). The Neuroticism subscale (BFI-N) measures the trait of neuroticism opposed to emotional stability, with increasing scores indicating a larger tendency to experience negative emotions. This version of the BFI has a Cronbach's alpha of 0.86 .

\section{AD symptoms and trajectories}

Our definition of "AD symptoms" was theoretically derived from the DSM-V definition of AD, which describes that AD symptoms are characterized by marked distress, while the distress should not meet criteria for another MD. Cutoff thresholds for the HADS have been established that are sensitive to detect any MD (score $\geq 11$ ) and depression (score $\geq 15$ )[43]. As such, we have assumed that a HADS score of 11 to 14 (i.e. marked distress but not depression) are indicative of $\mathrm{AD}$ symptoms. This is in line with previous studies reporting that the optimal HADS total score for screening for $\mathrm{AD}$ is 10 or $11[1,25,33]$. Thus, we predefined categories on HADS total: (1) $\leq 10$ was defined as "no MD symptoms", (2) 11 to 14 as "AD symptoms", and (3) $\geq 15$ as "any other MD symptoms". Trajectories were created based on HADS total over four assessments and defined as (a) no MD symptoms at all four assessments, (b) AD symptoms at all four assessments, (c) any other MD symptoms at all four assessments, and (d) fluctuating AD symptoms, i.e. an increase, decrease, or irregular pattern of HADS total.

\section{Data processing and statistical analyses}

Missing item scores on the HADS were replaced by the participants' subscale mean if at least four subscale items were answered [8]. Participants who completed the HADS all four assessments were considered completers, and participants who had a missing HADS or did not report date of birth or time since diagnosis were considered non-completers. Completers and non-completers were compared on demographic and clinical variables using t-tests for continuous variables and chi-square tests for categorical variables. Completers were assigned into our a priori defined trajectories based on their score above or below cut-off scores, and trajectories were compared on demographic and psychosocial variables with univariate testing (oneway ANOVA, chi-square tests, and post hoc analysis).
Variables that were significantly associated with trajectories membership were entered in a final multinominal regression analysis. Analyses were performed with SPSS version 25.

Latent class growth analysis (LCGA) was conducted using MPlus version 7 to identify data-based trajectories (classes) over time for HADS total, following the guidelines described by Jung and Wickrama [21]. By estimating individual differences (variability) in parameters reflecting participants' change in outcome over time, individuals are classified into latent classes based upon similar patterns in the outcome of interest (HADS). MPlus' full information maximum likelihood estimation for handling missing data was applied.

Following the guidelines, a single-class growth curve model was specified, as well as a three-class model. To determine the number of classes, the three-class model was compared with a two-class and four-class model, and the four-class model was compared with a three-class and five-class model. In total, the fit of five unconditional latent class models (i.e. models with no covariates) were estimated, with one to five linear classes. The number of classes was determined based on fit indices, model parsimony, and clinical interpretability. The model with the best fit has the smallest Bayesian Information Criterion (BIC) and significant $p$-values $(p<0.05)$ for the VuongLo-Mendell Rubin Likelihood Ratio Test (LMR-LRT) and the Bootstrap Likelihood Ratio Test (BLRT), which indicate that a model with a $k$ number of classes has a better fit than a model with k-1 number of classes. Other considerations were a higher entropy statistic (near 1.0), indicating the degree to which latent trajectories may be clearly distinguished, and higher posterior probabilities of group membership (near 1.0), indicating the degree to which individuals have been correctly classified into a class. For clinical interpretability, we also considered the number of participants (not less than $5 \%$ of total sample $(n \geq 23)$ ) of the identified classes. For each individual patient in the database, the predicted class of the best fitting model (i.e., with the optimal number of subgroups) was obtained.

\section{Results}

\section{Sample characteristics}

Of the 1205 eligible BCS who were invited, 459 participants $(38.1 \%)$ consented and completed the HADS at least once. Demographic and clinical variables of completers, non-completers, and the full sample are shown in Table 1 . Compared to non-completers, completers were older $(p=0.002)$ and had a lower education level $(p=0.026)$. 
Table 1 Socio-demographic and medical characteristics of participants

\begin{tabular}{|c|c|c|c|c|}
\hline & & $\begin{array}{l}\text { Completers } \\
N=293 \text { (valid \%) }\end{array}$ & $\begin{array}{l}\text { Non-completers } \\
N=166 \text { (valid \%) }\end{array}$ & $\begin{array}{l}\text { Full study sample } \\
N=459 \text { (valid \%) }\end{array}$ \\
\hline Dutch nationality & & $287(98.0 \%)$ & $162(98.8 \%)$ & $449(99.3 \%)$ \\
\hline Age (mean, years $\left(\mathrm{SD}^{\mathrm{a}} ;\right.$ range $\left.)\right)$ & & $57.8(9.3 ; 33.0-87.6)$ & $54.8(10.0 ; 33.2-83.8)$ & $56.7(9.7 ; 33.0-87.6)$ \\
\hline Marital status & & $228(78.4 \%)$ & $138(83.6 \%)$ & $366(80.3 \%)$ \\
\hline Children & & $240(82.5 \%)$ & $143(86.7 \%)$ & $383(84.0 \%)$ \\
\hline \multirow[t]{3}{*}{ Education } & Primary & $65(22.6 \%)$ & $24(14.7 \%)$ & $89(16.0 \%)$ \\
\hline & Secondary & $146(50.7 \%)$ & $78(47.9 \%)$ & $224(49.7 \%)$ \\
\hline & Tertiary & $77(26.7 \%)$ & $61(37.4 \%)$ & $138(30.6 \%)$ \\
\hline Time since diagnosis (mean, months) & & $33.1(\mathrm{SD} 16.1)$ & $33.1(\mathrm{SD} 16.1)$ & $33.3(\mathrm{SD} 16.0)$ \\
\hline Time since end of treatment (mean, months) & & $26.8(\mathrm{SD} 16.6)$ & $28.7($ SD 16.8$)$ & $28.6(\mathrm{SD} 16.7)$ \\
\hline Breast saving surgery & & $189(64.5 \%)$ & $98(60.1 \%)$ & $287(62.9 \%)$ \\
\hline Ablatio & & $39(13.3 \%)$ & $19(11.7 \%)$ & $58(12.7 \%)$ \\
\hline Breast amputation & & $75(25.6 \%)$ & $55(33.7 \%)$ & $130(28.5 \%)$ \\
\hline Chemotherapy & & $206(70.3 \%)$ & $123(75.0 \%)$ & $329(72.0 \%)$ \\
\hline Radiotherapy & & $226(77.4 \%)$ & $121(73.8 \%)$ & $347(76.1 \%)$ \\
\hline Hormone therapy & & $193(65.9 \%)$ & $102(62.2 \%)$ & $295(64.6 \%)$ \\
\hline Trastuzumab/Herceptin & & $37(12.7 \%)$ & $24(14.6 \%)$ & $61(13.4 \%)$ \\
\hline
\end{tabular}

${ }^{\mathrm{a}}$ Standard deviation

\section{Trajectories based on cut-off scores}

At group level, the average of all four HADS-assessments was $72.1 \%$ with no MD symptoms, $12.9 \%$ AD symptoms, and $15.0 \%$ any other MD symptoms. Classification in trajectories resulted in $157 \mathrm{BCS}(53.6 \%)$ in the trajectory no MD symptoms, 4 BCS (1.4\%) in the trajectory AD symptoms, $20 \mathrm{BCS}(6.8 \%)$ in the trajectory any other MD symptoms, and $112 \mathrm{BCS}(38.2 \%)$ in the trajectory fluctuating $\mathrm{AD}$ symptoms.

\section{Predictors of trajectories based on cut-off scores}

Given the low number of BCS in the trajectory AD symptoms, these BCS were merged with the trajectory fluctuating $\mathrm{AD}$ symptoms (stable\&fluc-AD symptoms) and compared to the trajectory no MD symptoms and trajectory any other MD symptoms on demographic and psychosocial characteristics at baseline (Table 2).

In univariate analyses, a difference between trajectories was observed for age $(p=0.041)$, previous psychological counselling $(p \leq 0.001)$, perceived social support discrepancy ( $p \leq 0.001)$, optimism $(p \leq 0.001)$, neuroticism $(p \leq 0.001)$, experience of a recent life event $(p<0.040)$, and being able to handle daily activities $(p=0.037)$.

The seven significant predictors were entered simultaneously in the multinominal logistic regression analysis with the trajectory no MD symptoms as the reference group (Table 2). The final model was statistically significant $\left(X^{2}=166.9, d f=14, p \leq 0.001\right.$, Cox \& Snell $\mathrm{R}^{2}=0.45$,
Nagelkerke $=0.54$, McFadden $=0.34)$. Experiencing a recent life event and previous psychological counselling did not contribute significantly to the overall statistical model (Table 2). Compared to BCS in the reference group, BCS in the trajectories stable\&fluc-AD symptoms and any other MD symptoms were less able to handle daily activities, perceived a larger social support discrepancy, and showed less optimism and more neuroticism. Additionally, BCS in the trajectory stable\&fluc-AD symptoms were younger compared to BCS in the reference group.

\section{Trajectories based on LCGA}

Using LCGA for the complete sample $(N=459)$, the intercept of the HADS total was 7.6 (95\% confidence interval [CI] 7.0-8.1, $p \leq 0.001$ ), which can be interpreted as no MD symptoms at baseline. There was a non-significant slope $(0.04 ; 95 \% \mathrm{CI}-0.11$ to $0.19, p=0.630)$, which can be interpreted as a stable HADS total during 1 year. The most appropriate choice based on fit indices, internal reliability, and interpretability was a three-class model (Table 3 ). The first trajectory consisted of 269 BCS (58.6\%) and was defined as "stable no MD symptoms" (low), as participants reported low baseline HADS total scores (intercept 3.60; 95\% CI 3.09-4.11) with a non-significant slope $(-0.09$ (95\% CI - 0.26-0.07)). The second trajectory was defined as "stable AD symptoms" (AD symptoms). For this trajectory of $151 \mathrm{BCS}(32.9 \%)$, the intercept was 11.38 (95\% CI 10.23-12.54) with a non-significant slope $(-0.1 ; 95 \%$ CI-0.39-0.19). The third trajectory was defined as "high 


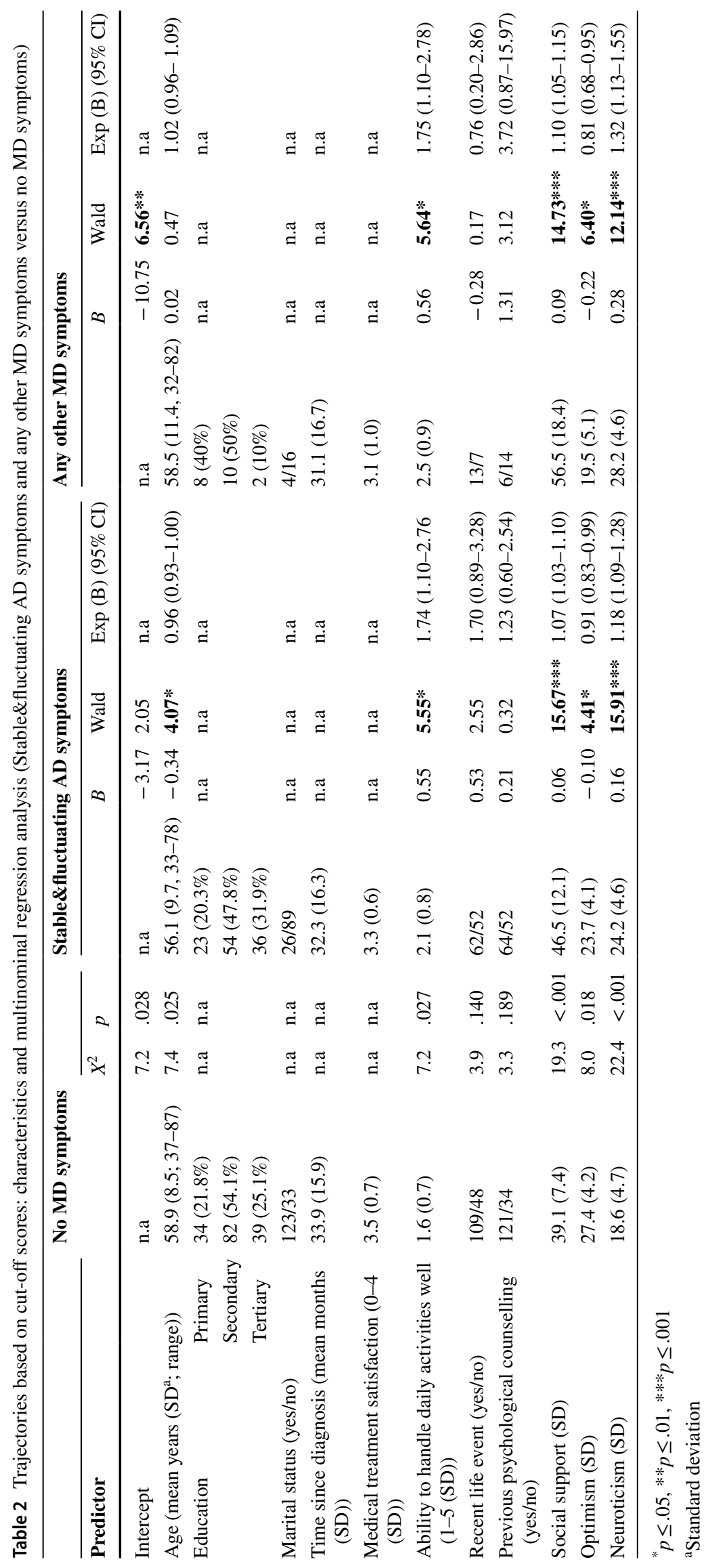


Table 3 Fit indices, entropy, and average posterior probabilities across models with different number of classes by HADS ${ }^{\mathrm{a}}$ score

\begin{tabular}{|c|c|c|c|c|c|c|c|c|}
\hline No. of classes & $\mathrm{BIC}^{\mathrm{b}}$ & LMR-LRT $^{\mathrm{c}}$ & BLR-T $^{\mathrm{d}}$ & Entropy & $n$ & $\begin{array}{l}\text { Posterior } \\
\text { probabilities }\end{array}$ & Intercept $\left(95 \% \mathrm{CI}^{\mathrm{e}}\right)$ & Slope linear $(95 \% \mathrm{CI})$ \\
\hline \multirow[t]{2}{*}{2} & 9684.567 & 0.0048 & 0.0000 & 0.885 & $350(76.3 \%)$ & 0.968 & $4.79(4.02-5.56)^{* * *}$ & $-0.06(-0.22-0.09)$ \\
\hline & & & & & $109(23.7 \%)$ & 0.953 & $15.68(13.81-17.55)^{* * *}$ & $0.29(-0.25-0.82)$ \\
\hline \multirow[t]{3}{*}{3} & 9328.060 & 0.0001 & 0.0000 & 0.880 & $269(58.6 \%)$ & 0.960 & $3.60(3.09-4.11)^{* * *}$ & $-0.09(-0.26-0.07)$ \\
\hline & & & & & $151(32.9 \%)$ & 0.918 & $11.38(10.23-12.54) * * *$ & $-0.10(-0.39-0.19)$ \\
\hline & & & & & $39(8.5 \%)$ & 0.938 & $19.83(17.54-22.12)^{* * *}$ & $1.29(0.30-2.29)^{*}$ \\
\hline \multirow[t]{4}{*}{4} & 9212.377 & 0.0200 & 0.0000 & 0.823 & $201(43.8 \%)$ & 0.916 & $2.59(2-3.18) * * *$ & $-0.07(-0.22-0.08)$ \\
\hline & & & & & $75(16.3 \%)$ & 0.903 & $14.04(12.74-15.33) * * *$ & $-0.14(-0.7-0.42)$ \\
\hline & & & & & $32(7.0 \%)$ & 0.951 & $20.50(18.12-22.88)^{* * *}$ & $1.48(0.36-2.6)^{* *}$ \\
\hline & & & & & $151(33.0 \%)$ & 0.847 & $7.90(6.7-9.1)^{* * *}$ & $-0.03(-0.37-0.31)$ \\
\hline \multirow[t]{5}{*}{5} & 9201.561 & 0.3348 & 0.0000 & 0.761 & $158(34.4 \%)$ & 0.892 & $2.09(1.66-2.51)^{* * *}$ & $-0.07(-0.21-0.07)$ \\
\hline & & & & & $48(10.4 \%)$ & 0.864 & $15.35(13.69-17.01)^{* * *}$ & $-0.26(-0.87-0.35)$ \\
\hline & & & & & $31(6.71 \%)$ & 0.942 & $20.52(18.04-23.01)^{* * *}$ & $1.53(0.36-2.7)^{* * *}$ \\
\hline & & & & & $133(29.0 \%)$ & 0.774 & $6.13(5.09-7.17) * * *$ & $-0.02(-0.4-0.35)$ \\
\hline & & & & & $89(19.4 \%)$ & 0.784 & $10.45(8.23-12.68)^{* * *}$ & $-0.05(-0.61-0.51)$ \\
\hline
\end{tabular}

${ }^{*} p \leq .05, * * p \leq .01, * * * p \leq .001$

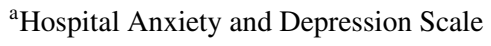

${ }^{\mathrm{b}}$ Bayesian Information Criterion

${ }^{\mathrm{c}}$ Vuong-Lo-Mendell Rubin Likelihood Ratio Test

${ }^{\mathrm{d}}$ Bootstrap Likelihood Ratio Test

${ }^{\mathrm{e}}$ Confidence interval

increasing any other MD symptoms" (high increasing). For this trajectory of $39 \mathrm{BCS}(8.5 \%)$, the intercept was 19.83 (95\% CI 17.54-22.12) with a significantly increasing slope (1.29; 95\% CI 0.30-2.29).

\section{Predictors of trajectories based on LCGA}

Univariate analyses (Table 4) showed differences between trajectories for age $(p=0.015)$, satisfaction with medical treatment $(p<0.004)$, being able to handle daily activities ( $p<0.003)$, previous psychological counselling $(p \leq 0.001)$, experiencing a recent life event $(p=0.010)$, social support discrepancy $(p \leq 0.001)$, optimism $(p \leq 0.001)$ and neuroti$\operatorname{cism}(p \leq 0.001)$.

These eight predictors were included in the final model with the low trajectory as reference group. The final model was statistically significant $\left(\chi^{2}=264.5, d f=16, p \leq 0.001\right.$, Cox \& Snell $\mathrm{R}^{2}=0.46$, Nagelkerke $=0.55$, McFadden $=0.35$ ). Age, ability to handle daily activities, social support discrepancy, neuroticism, and optimism contributed significantly to the statistical model. BCS in the AD symptoms trajectory and the high increasing trajectory were less able to handle daily activities, perceived a larger social support discrepancy, and showed more neuroticism compared to $\mathrm{BCS}$ in the low trajectory. BCS in the AD symptoms trajectory were younger compared to BCS in the low trajectory, and BCS in the high increasing trajectory reported less optimism compared to BCS in the low trajectory.

\section{Discussion}

In this study, three distinct 1-year trajectories in HADS scores were found in BCS using two different statistical approaches: one approach with clinical cut-off scores to indicate AD symptoms or MD symptoms and one data-driven approach to predict classes of BCS with a similar course of AD symptoms or MD symptoms. The "low" trajectory was found in more than half of the BCS. The second trajectory with (fluctuating) AD symptoms was found in about one-third of the BCS. The trajectory with (high increasing) any other MD symptoms was found in fewer than one in ten BCS. Furthermore, the approach based on cut-off scores showed a very low (1.4\%) percentage of BCS with stable AD symptoms and fluctuating scores below and above cutoff scores in almost $40 \%$ of the participants. With the latent modelling approach, we found a trajectory AD symptoms in one-third of the BCS, with a wide confidence interval of HADS scores per assessment and no significant change over time. Thus, both statistical approaches showed that $\mathrm{AD}$ symptoms can fluctuate in a significant proportion of BCS over time and that a pattern of stable AD symptoms was 


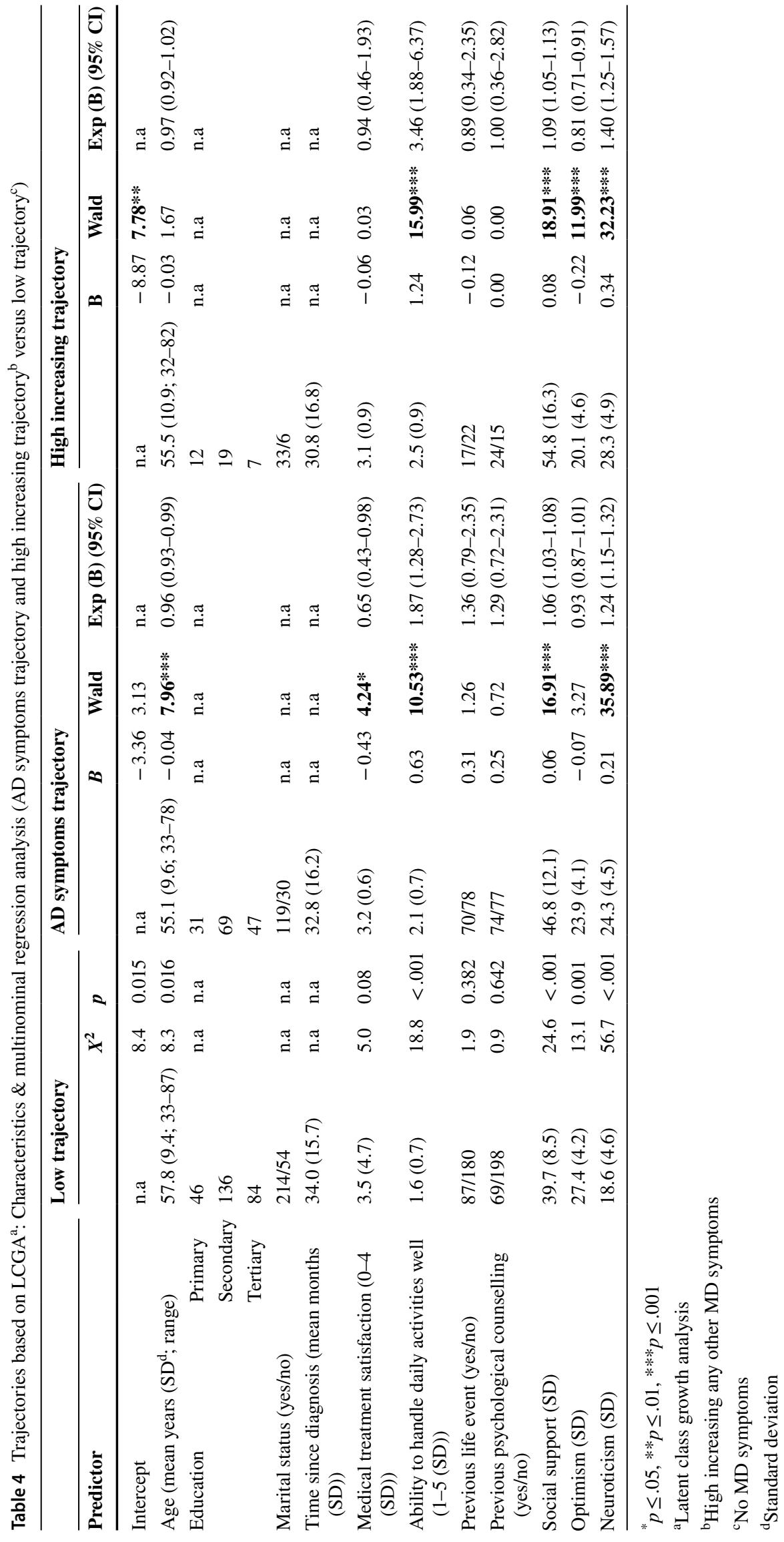


not present in this sample. This questions the validity of the diagnosis $\mathrm{AD}$ in $\mathrm{BCS}$.

The detection of the low trajectories and (high increasing) any other MD symptoms is in line with previous trajectory studies $[17,18,22,26,27]$. These studies all reported trajectories as "resilient" and "chronic", with a stable course of few and high symptoms post-treatment up to 6 months [18, 22, 27], 8 months [26], and 4 years [17]. Non-stable trajectories were observed in all previous studies as well. Our study provided additional detailed observations by means of multiple assessments within a 1-year period, indicating more individual fluctuations in $\mathrm{AD}$ symptoms than was expected based on earlier findings. Fluctuations in AD symptoms were found independent of time since diagnosis, which is not in line with the DSM-V definition of AD [3] which assumes that $\mathrm{AD}$ diminishes over time, implying a self-healing process [11]. The discrepancies between this study and the established AD criteria stress the debate of $\mathrm{AD}$ diminishing after 6 months after AD symptom occurrence or becoming persistent in case of ongoing stressors in the cancer survivor context. Therefore, future research could be directed towards exploring acute and persistent $\mathrm{AD}$ immediately post-diagnosis and whether symptoms might fluctuate over time.

Compared to BCS in the trajectories with no MD symptoms, characteristics of BCS in the trajectories of AD symptoms or MD symptoms were a larger social support discrepancy, less optimism, and more neuroticism. These findings are in line with previous trajectory studies, where less social support [17, 27], less optimism [18, 26], and higher scores on neuroticism $[18,26]$ were observed in "chronic distress" or "lower mental functioning" trajectories. Lower ability to handle daily activities is in line to the criteria of $\mathrm{AD}$ [3], where poorer functioning in social relations, work, or study is observed in people who are diagnosed with AD. Lastly, with the exception of Lam et al. [26], previous trajectory studies did not find age differences between trajectories, which is contradictory to our study. BCS with a trajectory of (fluctuating) AD symptoms were almost 3 years younger compared to BCS belonging to the trajectory no MD symptoms. A systematic review including cross-sectional and longitudinal studies found that a younger age increased the risk of distress [40]. These previous findings regarding predictors combined with the results of our study emphasize the relevance for clinicians to monitor these predictors to detect vulnerable BCS showing AD symptoms.

\section{Study limitations}

The results should be interpreted carefully because of selection bias in the study sample. Participants who completed all questionnaires were older and lower educated compared to participants who did not complete all questionnaires, although the sample used for the cut-off score analysis was comparable to the sample in the LCGA analysis. Furthermore, analysis of the predictive value of education was not possible due to too small cells, resulting in inconclusive findings on education. In our study, we assessed whether participants had previously received psychological counselling. We did not, however, assess whether participants had a history of mental illness, which could have been an important predictor to developing AD. This study was an additional analysis of a dataset on the course of fear of cancer recurrence (FCR) over time in BCS [15]. Secondary analyses reduce research participation burden, but results might be less generalizable to the overall BCS population, since BCS signed up for research investigating FCR instead of $\mathrm{AD}$ related to cancer.

For research purposes, the analyses of the HADS are of great value to gain insight in which BCS are at risk for AD. The HADS, however, does not assess impairments in social or occupational functioning, which is a limitation. While not assessed thoroughly in our study, BCS with a trajectory of (fluctuating) AD symptoms reported less ability to handle daily activities and had a larger perceived social support discrepancy. A diagnostic interview, use of an AD-specific questionnaire, or combining measures would capture $\mathrm{AD}$ more accurately. Finally, due to the small number of participants with AD symptoms, we were not able to further categorize subtypes of AD.

\section{Clinical implications}

This study used two different approaches to analyse the data, combining methodologies used in previous studies to observe the course of AD symptoms: a clinical point of view in using a cut-off score to screen for a possible $\mathrm{AD}$ or $\mathrm{MD}$ and a statistical point of view to predict latent classes based on scores over time. Both methodologies detected fluctuating symptoms over time. This would imply that conclusions based on single assessment HADS scores in clinical practice would not be sensitive enough to detect those patients with $\mathrm{AD}$ symptoms and for whom a diagnosis of $\mathrm{AD}$ might be applicable.

\section{Conclusion}

A substantial proportion of BCS up to 5 years post-diagnosis showed fluctuating AD symptoms, and only a negligible percentage of the cases had a stable course of AD symptoms. We suggest handling single assessment cut-off scores with caution. 
Author contribution José Custers and Judith Prins contributed to the study conception and design. José Custers performed the data collection. Material preparation and analysis were performed by all authors. All authors wrote and commented on the first and following drafts of the manuscript. All authors read and approved the final manuscript.

Funding This work was supported by Pink Ribbon, The Netherlands, Grant No. 2011.WO11.C106.

Availability of data and material (data transparency) Data are available upon reasonable request. Requests to access the datasets should be directed to jose.custers@ radboudumc.nl.

Code availability Not applicable.

\section{Declarations}

Ethics approval All procedures performed in studies involving human participants were in accordance with the ethical standards of the institutional and/or national research committee and with the 1964 Helsinki Declaration and its later amendments or comparable ethical standards. The local Ethics Committee approved the study (CMO Regio ArnhemNijmegen 2012/227).

Consent to participate Informed consent was obtained from all individual participants included in the study.

Consent for publication Patients signed informed consents acknowledging that their data would be used for research publication.

Conflict of interest The authors declare no competing interests.

Disclaimer The study sponsor had no role in the study design, data collection, analysis and interpretation of data, writing of the report, or decision to submit the paper for publication.

Open Access This article is licensed under a Creative Commons Attribution 4.0 International License, which permits use, sharing, adaptation, distribution and reproduction in any medium or format, as long as you give appropriate credit to the original author(s) and the source, provide a link to the Creative Commons licence, and indicate if changes were made. The images or other third party material in this article are included in the article's Creative Commons licence, unless indicated otherwise in a credit line to the material. If material is not included in the article's Creative Commons licence and your intended use is not permitted by statutory regulation or exceeds the permitted use, you will need to obtain permission directly from the copyright holder. To view a copy of this licence, visit http://creativecommons.org/licenses/by/4.0/.

\section{References}

1. Akizuki N, Akechi T, Nakanishi T, Yoshikawa E, Okamura M, Nakano T, Murakami Y, Uchitomi Y (2003) Development of a brief screening interview for adjustment disorders and major depression in patients with cancer. Cancer 97:2605-2613. https:// doi.org/10.1002/cncr.11358

2. American Cancer Society (2020) Cancer facts \& figures 2020. In: Editor (ed)^(eds) Book Cancer Facts \& Figures 2020. American Cancer Society City
3. American Psychiatric Association (2013) Diagnostic and statistical manual of mental disorders, 5th edition (DSM-5). American Psychiatric Association, Washington, DC

4. Andrykowski MA, Cordova MJ, Studts JL, Miller TW (1998) Posttraumatic stress disorder after treatment for breast cancer: prevalence of diagnosis and use of the PTSD checklist - Civilian version (PCL-C) as a screening instrument. J Consult Clin Psych 66:586-590. https://doi.org/10.1037/0022-006x.66.3.586

5. Armes J, Crowe M, Colbourne L, Morgan H, Murrells T, Oakley C, Palmer N, Ream E, Young A, Richardson A (2009) Patients' supportive care needs beyond the end of cancer treatment: a prospective, longitudinal survey. J Clin Oncol 27:6172-6179. https:// doi.org/10.1200/JCO.2009.22.5151

6. Bachem R, Casey P (2018) Adjustment disorder: a diagnosis whose time has come. J Affect Disord 227:243-253. https://doi. org/10.1016/j.jad.2017.10.034

7. Beck AT, Steer RA, Brown GK (1996) Beck depression inventory-II. San Antonio 78:490-498

8. Bell ML, Fairclough DL, Fiero MH, Butow PN (2016) Handling missing items in the Hospital Anxiety and Depression Scale (HADS): a simulation study. BMC Res Notes 9:479. https://doi. org/10.1186/s13104-016-2284-Z

9. Carta MG, Balestrieri M, Murru A, Hardoy MC (2009) Adjustment disorder: epidemiology, diagnosis and treatment. Clin Pract Epidemiology Ment Health 5:15. https://doi.org/10.1186/ 1745-0179-5-15

10 Casey P (2009) Adjustment disorder: epidemiology, diagnosis and treatment. CNS Drugs 23:927-938. https://doi.org/10.2165/ 11311000-000000000-00000

11. Casey P, Bailey S (2011) Adjustment disorders: the state of the art. World Psychiatry 10:11-18. https://doi.org/10.1002/j.20515545.2011.tb00003.x

12. Cook SA, Salmon P, Hayes G, Byrne A, Fisher PL (2018) Predictors of emotional distress a year or more after diagnosis of cancer: a systematic review of the literature. Psychooncology 27:791-801. https://doi.org/10.1002/pon.4601

13. Cornelius LR, Brouwer S, de Boer MR, Groothoff JW, van der Klink JJ (2014) Development and validation of the diagnostic interview adjustment disorder (DIAD). Int J Methods Psychiatr Res 23:192-207. https://doi.org/10.1002/mpr.1418

14. Costanzo ES, Lutgendorf SK, Mattes ML, Trehan S, Robinson CB, Tewfik F, Roman SL (2007) Adjusting to life after treatment: distress and quality of life following treatment for breast cancer. Br J Cancer 97:1625-1631. https://doi.org/10.1038/sj. bjc.6604091

15. Custers JA, Kwakkenbos L, van der Graaf WT, Prins JB, Gielissen MF, Thewes B (2020) Not as stable as we think: a descriptive study of 12 monthly assessments of fear of cancer recurrence among curatively-treated breast cancer survivors 0-5 years after surgery. Front Psychol 11:580979. https://doi.org/10. 3389/fpsyg.2020.580979

16. Denissen JJ, Geenen R, van Aken MA, Gosling SD, Potter J (2008) Development and validation of a Dutch translation of the Big Five Inventory (BFI). J Pers Assess 90:152-157. https://doi. org/10.1080/00223890701845229

17 Helgeson VS, Snyder P, Seltman H (2004) Psychological and physical adjustment to breast cancer over 4 years: identifying distinct trajectories of change. Health Psychol 23:3-15. https:// doi.org/10.1037/0278-6133.23.1.3

18. Henselmans I, Helgeson VS, Seltman H, de Vries J, Sanderman $\mathrm{R}$, Ranchor AV (2010) Identification and prediction of distress trajectories in the first year after a breast cancer diagnosis. Health Psychol 29:160-168. https://doi.org/10.1037/a0017806

19. Hund B, Reuter K, Harter M, Brahler E, Faller H, Keller M, Schulz H, Wegscheider K, Weis J, Wittchen HU, Koch U, Friedrich M, Mehnert A (2016) Stressors, symptom profile, and 
predictors of adjustment disorder in cancer patients. Results from an Epidemiological Study with the Composite International Diagnostic Interview, Adaptation for Oncology (Cidi-O). Depress Anxiety 33:153-161. https://doi.org/10.1002/da.22441

20 Ibbotson T, Maguire P, Selby P, Priestman T, Wallace L (1994) Screening for anxiety and depression in cancer-patients - the effects of disease and treatment. Eur J Cancer 30:37-40. https:// doi.org/10.1016/S0959-8049(05)80015-2

21. Jung T, Wickrama KAS (2008) An introduction to latent class growth analysis and growth mixture modeling. Soc Personal Psychol Compass 2:302-317. https://doi.org/10.1111/j.17519004.2007.00054.x

22. Kant J, Czisch A, Schott S, Siewerdt-Werner D, Birkenfeld F, Keller M (2018) Identifying and predicting distinct distress trajectories following a breast cancer diagnosis - from treatment into early survival. J Psychosom Res 115:6-13. https://doi.org/ 10.1016/j.jpsychores.2018.09.012

23. Kissane DW, Grabsch B, Love A, Clarke DM, Bloch S, Smith GC (2004) Psychiatric disorder in women with early stage and advanced breast cancer: a comparative analysis. Aust N Z J Psychiatry 38:320-326. https://doi.org/10.1080/j.1440-1614.2004. 01358.x

24. Kostev K, Jacob L, Kalder M (2017) Risk of depression, anxiety, and adjustment disorders in women with a suspected but unconfirmed diagnosis of breast or genital organ cancer in Germany. Cancer Causes Control 28:1021-1026. https://doi.org/10.1007/ s10552-017-0948-1

25. Kugaya A, Akechi T, Okuyama T, Okamura H, Uchitomi Y (1998) Screening for psychological distress in Japanese cancer patients. Jpn J Clin Oncol 28:333-338. https://doi.org/10.1093/jjco/28.5. 333

26. Lam WW, Bonanno GA, Mancini AD, Ho S, Chan M, Hung WK, Or A, Fielding R (2010) Trajectories of psychological distress among Chinese women diagnosed with breast cancer. Psychooncology 19:1044-1051. https://doi.org/10.1002/pon.1658

27 Lotfi-Jam K, Gough K, Schofield P, Aranda S, Jefford M (2019) A longitudinal study of four unique trajectories of psychological distress in cancer survivors after completing potentially curative treatment. Acta Oncol 58:782-789. https://doi.org/10.1080/02841 86X.2018.1562209

28. Maercker A, Einsle F, Kollner V (2007) Adjustment disorders as stress response syndromes: a new diagnostic concept and its exploration in a medical sample. Psychopathology 40:135-146

29. Mehnert A, Brähler E, Faller H, Härter M, Keller M, Schulz H, Wegscheider K, Weis J, Boehncke A, Hund B (2014) Four-week prevalence of mental disorders in patients with cancer across major tumor entities. J Clin Oncol 32:3540-3546. https://doi.org/ 10.1200/JCO.2014.56.0086

30. Mehnert A, Koch U (2007) Prevalence of acute and post-traumatic stress disorder and comorbid mental disorders in breast cancer patients during primary cancer care: a prospective study. PsychoOncol 16:181-188. https://doi.org/10.1002/pon.1057

31. Mitchell AJ, Chan M, Bhatti H, Halton M, Grassi L, Johansen C, Meader N (2011) Prevalence of depression, anxiety, and adjustment disorder in oncological, haematological, and palliative-care settings: a meta-analysis of 94 interview-based studies. Lancet Oncol 12:160-174. https://doi.org/10.2165/11311000-00000 0000-00000
32. Nederlandse Kankerregistratie (NKR) I (2020). In: Editor (ed)^(eds) Book, City

33. Ozalp E, Soygur H, Cankurtaran E, Turhan L, Akbiyik D, Geyik P (2008) Psychiatric morbidity and its screening in Turkish women with breast cancer: a comparison between the HADS and SCID tests. Psychooncology 17:668-675. https://doi.org/10.1002/pon. 1286

34. Riba MB, Donovan KA, Andersen B, Braun I, Breitbart WS, Brewer BW, Buchmann LO, Clark MM, Collins M, Corbett C (2019) Distress management, version 3.2019, NCCN clinical practice guidelines in oncology. J Natl Compr Canc Netw 17:1229 1249. https://doi.org/10.6004/jnccn.2019.0048

35 Scheier MF, Carver CS (1985) Optimism, coping, and health: assessment and implications of generalized outcome expectancies. Health Psychol 4:219-247. https://doi.org/10.1037//0278-6133.4. 3.219

36 Shevlin M, Hyland P, Ben-Ezra M, Karatzias T, Cloitre M, Vallieres F, Bachem R, Maercker A (2020) Measuring ICD-11 adjustment disorder: the development and initial validation of the International Adjustment Disorder Questionnaire. Acta Psychiatr Scand 141:265-274. https://doi.org/10.1111/acps.13126

37. Spinhoven P, Ormel J, Sloekers PP, Kempen GI, Speckens AE, Van Hemert AM (1997) A validation study of the Hospital Anxiety and Depression Scale (HADS) in different groups of Dutch subjects. Psychol Med 27:363-370. https://doi.org/10.1017/s0033 291796004382

38. Stanton AL (2012) What happens now? Psychosocial care for cancer survivors after medical treatment completion. J Clin Oncol 30:1215-1220. https://doi.org/10.1200/JCO.2011.39.7406

39. Stevens L, Rodin I (2011) Reactions to stress. In: Stevens L, Rodin I (eds) Psychiatry (Second Edition). Churchill Livingstone, pp. 62-63. https://doi.org/10.1016/B978-0-7020-3396-4.00035-4

40. Syrowatka A, Motulsky A, Kurteva S, Hanley JA, Dixon WG, Meguerditchian AN, Tamblyn R (2017) Predictors of distress in female breast cancer survivors: a systematic review. Breast Cancer Res Treat 165:229-245. https://doi.org/10.1007/ s10549-017-4290-9

41. Tang HY, Xiong HH, Deng LC, Fang YX, Zhang J, Meng H (2020) Adjustment disorder in female breast cancer patients: prevalence and its accessory symptoms Curr. Med Sci 40:510-517. https://doi.org/10.1007/s11596-020-2205-1

42. van Sonderen FLP (2012) Het meten van de sociale steun met de sociale steunlijst-interacties (SSL-I) en sociale steunlijst-discrepanties (SSL-D): een handleiding. UMCG / Rijksuniversiteit Groningen, Research Institute SHARE Groningen

43. Vodermaier A, Millman RD (2011) Accuracy of the Hospital Anxiety and Depression Scale as a screening tool in cancer patients: a systematic review and meta-analysis. Support Care Cancer 19:1899-1908. https://doi.org/10.1007/s00520-011-1251-4

Publisher's Note Springer Nature remains neutral with regard to jurisdictional claims in published maps and institutional affiliations. 